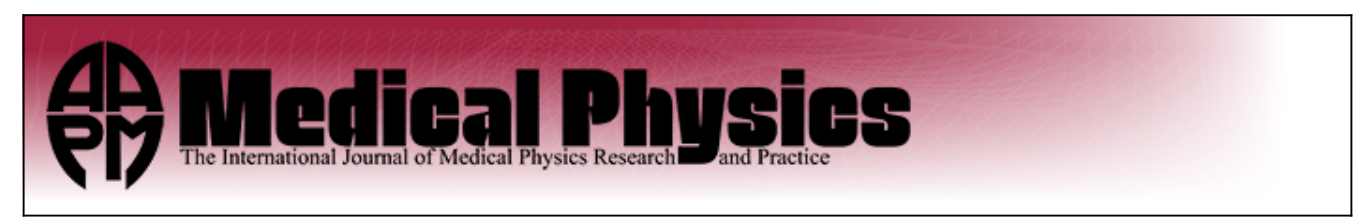

\title{
Evaluation of a synthetic single-crystal diamond detector for relative dosimetry on the Leksell Gamma Knife Perfexion radiosurgery system
}

Pietro Mancosu, Giacomo Reggiori, Antonella Stravato, Anna Gaudino, Francesca Lobefalo, Valentina Palumbo, Piera Navarria, Anna Ascolese, Piero Picozzi, Marco Marinelli, Gianluca Verona-Rinati, Stefano Tomatis, and Marta Scorsetti

Citation: Medical Physics 42, 5035 (2015); doi: 10.1118/1.4927569

View online: http://dx.doi.org/10.1118/1.4927569

View Table of Contents: http://scitation.aip.org/content/aapm/journal/medphys/42/9?ver=pdfcov

Published by the American Association of Physicists in Medicine

\section{Articles you may be interested in}

A novel synthetic single crystal diamond device for in vivo dosimetry

Med. Phys. 42, 4636 (2015); 10.1118/1.4926556

A synthetic diamond diode in volumetric modulated arc therapy dosimetry

Med. Phys. 40, 092103 (2013); 10.1118/1.4818256

Gamma Knife output factor measurements using VIP polymer gel dosimetry

Med. Phys. 36, 4277 (2009); 10.1118/1.3183500

Unintended attenuation in the Leksell Gamma Knife ${ }^{\circledR}$ Perfexion ${ }^{\mathrm{TM}}$ calibration-phantom adaptor and its effect on dose calibration

Med. Phys. 36, 1208 (2009); 10.1118/1.3093240

GAFChromic film dosimetry with a flatbed color scanner for Leksell Gamma Knife therapy

Med. Phys. 31, 1243 (2004); 10.1118/1.1712393

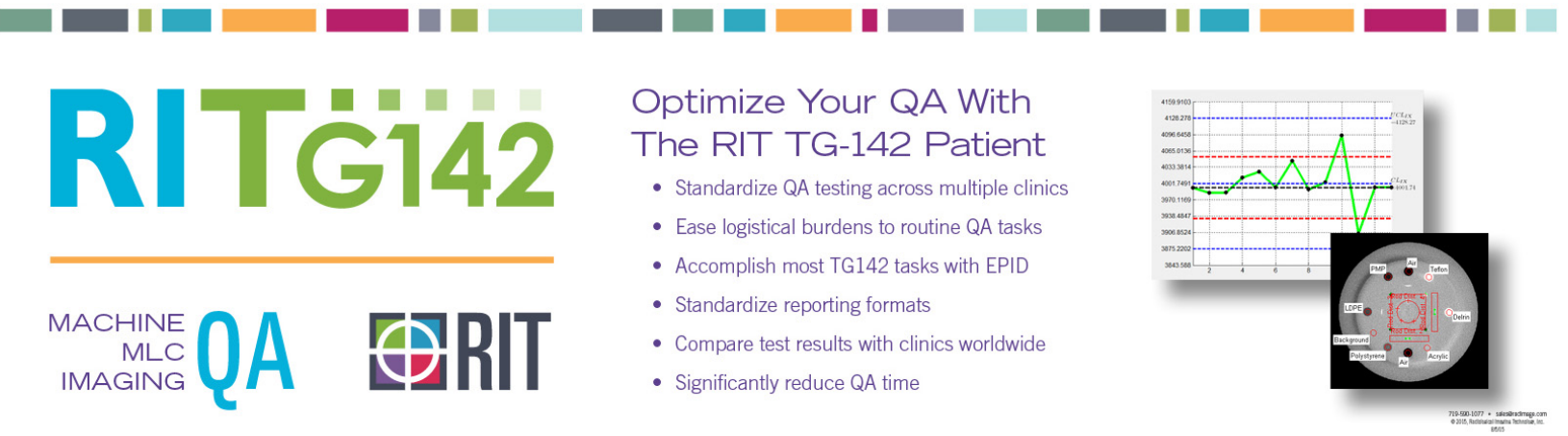




\title{
Evaluation of a synthetic single-crystal diamond detector for relative dosimetry on the Leksell Gamma Knife Perfexion radiosurgery system
}

\author{
Pietro Mancosu, Giacomo Reggiori, ${ }^{a}$ Antonella Stravato, Anna Gaudino, \\ Francesca Lobefalo, and Valentina Palumbo \\ Physics Service of Radiation Oncology Department, Clinical and Research Center, Rozzano, Milan 20098, Italy \\ Piera Navarria and Anna Ascolese \\ Radiation Oncology Department, Humanitas Clinical and Research Center, Rozzano, Milan 20089, Italy
}

Piero Picozzi

Neurosurgery Department, Humanitas Clinical and Research Center, Rozzano, Milan 20089, Italy

Marco Marinelli and Gianluca Verona-Rinati

Dipartimento di Ingegneria Industriale, Università di Roma Tor Vergata, Roma 00133, Italy

Stefano Tomatis

Physics Service of Radiation Oncology Department, Clinical and Research Center, Rozzano, Milan 20098, Italy

Marta Scorsetti

Radiation Oncology Department, Humanitas Clinical and Research Center, Rozzano, Milan 20089, Italy

(Received 18 July 2014; revised 16 June 2015; accepted for publication 14 July 2015;

published 5 August 2015)

Purpose: To evaluate the new commercial PTW-60019 synthetic single-crystal microDiamond detector (PTW, Freiburg, Germany) for relative dosimetry measurements on a clinical Leksell Gamma Knife Perfexion radiosurgery system.

Methods: Detector output ratios (DORs) for 4 and $8 \mathrm{~mm}$ beams were measured using a microDiamond (PTW-60019), a stereotactic unshielded diode [IBA stereotactic field detector (SFD)], a shielded diode (IBA photon field detector), and GafChromic EBT3 films. Both parallel and transversal acquisition directions were considered for PTW-60019 measurements. Measured DORs were compared to the new output factor reference values for Gamma Knife Perfexion ( 0.814 and 0.900 for 4 and $8 \mathrm{~mm}$, respectively). Profiles in the three directions were also measured for the $4 \mathrm{~mm}$ beam to evaluate full width at half maximum (FWHM) and penumbra and to compare them with the corresponding Leksell GammaPlan profiles.

Results: FWHM and penumbra for PTW-60019 differed from the calculated values by less than 0.2 and $0.3 \mathrm{~mm}$, for the parallel and transversal acquisitions, respectively. GafChromic films showed FWHM and penumbra within $0.1 \mathrm{~mm}$. The output ratio obtained with the PTW-60019 for the $4 \mathrm{~mm}$ field was $1.6 \%$ greater in transverse direction compared to the nominal value. Comparable differences up to $0.8 \%$ and $1.0 \%$ for, respectively, GafChromic films and SFD were found.

Conclusions: The microDiamond PTW-60019 is a suitable detector for commissioning and routine use of Gamma Knife with good agreement of both DORs and profiles in the three directions. (C) 2015 American Association of Physicists in Medicine. [http://dx.doi.org/10.1118/1.4927569]

Key words: single microDiamond detector, small field dosimetry, Gamma Knife Perfexion, output factor

\section{INTRODUCTION}

Leksell Gamma Knife Perfexion (LGKP) is the latest model of the gamma stereotactic radiosurgery system that enhances the potential of previous Leksell Gamma Knife (LGK) models. In this version, the $192{ }^{60} \mathrm{Co}$ sources are divided in eight independent sectors, each sector can be placed in four positions (beam off, 4, 8, and $16 \mathrm{~mm}$ ), allowing 65536 different possible arrangements for each shot. ${ }^{1,2}$

As with the previous LGK models, the absolute output (dose rate) of the largest open collimator (i.e., $16 \mathrm{~mm}$ ) is calibrated by reference dosimetry and the other collimators (i.e., 4 and $8 \mathrm{~mm}$ ) are modeled into the treatment planning system according to the output factor (OF) values. In this context, the new Leksell GammaPlan 10 (LGP) introduces the dose calculation algorithm TMR 10, which is an evolution of the water-based algorithm available in previous versions. In particular, improved Monte Carlo (MC) simulations have resulted in new values of OFs for LGP. The new reference values are 0.900 and 0.814 for 8 and $4 \mathrm{~mm}$, respectively, and replace the old reference values (0.924 and 0.805) (Data from the ELEKTA White Paper 2012. "A new TMR dose algorithm in Leksell GammaPlan.").

The verification of these new values is essential despite the difficulties associated with the dosimetry of small radiosurgical beams being well known. The change of the physical 
conditions due to the loss of lateral electronic equilibrium and the various perturbations (e.g., volume averaging artifacts, detector density response, and other minor corrections) makes these measurements complicated. According to the new small field formalism introduced by Alfonso et al. ${ }^{3}$ an output factor can be defined only when ratios of absolute dose measurements (or Monte Carlo calculations) are considered. When the ratio of readings is not corrected by the $k_{Q_{\text {clin }} \cdot Q_{\text {msr }}}^{f_{\text {cli }} \cdot \text { factor as }}$ introduced in Ref. 3, OFs cannot be defined. To avoid ambiguities and be consistent throughout the paper, we will use the detector output ratio (DOR) terminology when referring to ratio of readings that cannot be strictly considered OFs.

It is known that silicon diode detectors exhibit an overresponse to low-energy photon scatter. The relative contribution of this scatter increases with field size, and for regular field sizes, shielding has been used to compensate for this over-response. ${ }^{4,5}$ The shield, however, increases the detector size and, in small fields, introduces perturbations. Unshielded detectors are thus more appropriate for small field dosimetry.

Film dosimetry, especially with GafChromic films (GAFs), was shown to be a reasonable choice for performing dosimetric measurements in small beams. ${ }^{6-8}$ In fact, films remove the difficulty of using finite size detectors. Since the spatial resolution is of the order of several micrometers, the overall measurement resolution is generally limited by the scanner. Films also provide 2D dose maps. Many studies have been carried out in the past for the other LGK models. ${ }^{5,9-14}$ When performing film dosimetry, however, transition dose must be accounted of. This dose has to be evaluated independently with another device, typically a small field detector. For this reasons, investigating the possibility of using a single instrument, as a microDiamond detector, for the whole measurement process is of primary interest.

The purpose of this study was thus to measure the detector output ratios for 4 and $8 \mathrm{~mm}$ collimators for the LGKP unit using the new synthetic single-crystal diamond detector of PTW and other classical dosimeters comparing the measured values with the effective OFs provided by ELEKTA. The characteristics of the synthetic diamond detector (PTW-60019), such as small active volume $\left(0.004 \mathrm{~mm}^{3}\right)$, near water-equivalence $\left(Z_{\text {eff }}=6\right)$, and low energy dependence, make it an interesting candidate for small field dose measurements as demonstrated by many authors. ${ }^{15-19}$ To our knowledge, no one demonstrated the feasibility of using this synthetic diamond for OFs measurement for LGKP. Profiles in the three directions were also measured for the $4 \mathrm{~mm}$ collimator.

\section{METHODS AND MATERIALS}

\section{A. Detectors and films}

The measurements were performed using the new microDiamond PTW-60019, a stereotactic unshielded diode [IBA stereotactic field detector (SFD)], a shielded diode [IBA photon field detector (PFD)], and Radiochromic EBT3 films (GafChromic, ISP Technology, Wayne, NJ). Main characteristics are reported in Table I.

\section{A.1. MicroDiamond PTW-60019}

The microDiamond PTW-60019 is a commercial synthetic single-crystal diamond detector, in Schottky diode configuration operating with zero bias voltage. ${ }^{15-18}$ The microDiamond active volume is composed of a $1 \mu \mathrm{m}$ thick intrinsic diamond layer, with a $1 \mathrm{~mm}$ radius and a total sensitive volume of $0.004 \mathrm{~mm}^{3}$. The effective point of measurement is $1 \mathrm{~mm}$ from the housing surface. MicroDiamond signal was detected by the DOSE-1 electrometer (IBA Dosimetry). Detector response was measured in $\mathrm{nC}$ over a time of 60 s (i.e., around $3.2 \mathrm{~Gy}$ ). Profiles were acquired with a spacing of $0.5-1.0 \mathrm{~mm}$.

\section{A.2. Diodes}

The PFD (IBA Dosimetry, Schwarzenbruck, Germany) is a p-type shielded silicon diode whose characteristics in terms of size, electronic density, and sensitivity are reported in Table I. The $2 \mathrm{~mm}$ wide and $0.03 \mathrm{~mm}$ thick active volume is surrounded by a thin epoxy resin layer that brings the effective measurement point $\approx 0.5 \mathrm{~mm}$ under the surface. The PFD was considered for DOR comparison with PTW-60019 because of their comparable active diameters.

The SFD (IBA Dosimetry, Schwarzenbruck, Germany) is a very small semiconductor detector constructed for relative dosimetry based on highly doped p-type silicon. The frontal active surface is $\approx 0.3 \mathrm{~mm}^{2}$ allowing a very high spatial resolution. The absence of a shielding, however, leads to an overestimation of the low-energy doses due to its strong energy dependence.

TABLE I. Detectors and film characteristics.

\begin{tabular}{llccccc}
\hline \hline Detector & \multicolumn{1}{c}{ Type } & $\begin{array}{c}\text { Active volume } \\
\left(\mathrm{mm}^{3}\right)\end{array}$ & $\begin{array}{c}\text { Active } \\
\text { diameter }(\mathrm{mm})\end{array}$ & Material & $Z_{\text {eff }}$ & $\begin{array}{c}\text { Nominal sensitivity } \\
(\mathrm{nC} / \mathrm{Gy})\end{array}$ \\
\hline PTW-60019 & $\begin{array}{l}\text { Synthetic } \\
\text { diamond }\end{array}$ & 0.004 & 2 & Diamond & 6 & $0.7-1.2$ \\
SFD & $\begin{array}{l}\text { Unshielded } \\
\text { diode } \\
\text { Shielded } \\
\text { PFD }\end{array}$ & 0.017 & 0.188 & Silicon & 14 & 6 \\
EBT3 & $\begin{array}{l}\text { Radiochromic } \\
\text { films }\end{array}$ & - & 2 & Silicon & 14 & 33 \\
\hline \hline
\end{tabular}




\section{A.3. Film-GafChromics}

Radiochromic EBT3 films (GafChromic, ISP Technology, Wayne, NJ) were used for film measurements. The standard method of relating the film response to the dose is via the dose-exposure calibration curve. The GafChromic films were calibrated on Gamma Knife (i.e., using ${ }^{60} \mathrm{Co}$ ) in the range 0-6 Gy, with $1 \mathrm{~Gy}$ spacing, using the $16 \mathrm{~mm}$ aperture. The points were fitted with a third grade polynomial function provided by the software (IBA Dosimetry ${ }^{\circledR}$ OmniPro-I'mRT). All measured films were scanned using a 48 bit scanner (Epson Expression 1000XL, Epson America, Sunnyvale, CA) with a resolution of $72 \mathrm{dpi}$, as reported in the literature, ${ }^{20}$ yielding a pixel resolution of less than $0.4 \mathrm{~mm}$. The images were scanned using the red channel. The film images were analyzed using the IBA Dosimetry ${ }^{\circledR}$ OmniPro-I'mRT software. Individual film samples were always placed exactly at the same scanning position to exclude any variation in the scanner response over scan field. All samples were scanned in the same orientation as recommended by the film manufacturer. Square ROI of $4 \times 4$ pixels (i.e., $1.4 \times 1.4 \mathrm{~mm}$ ) was used for all $\mathrm{GafChromic}$ films' evaluations (also for OF estimation). Output factors were measured by irradiating the GafChromic films for $1 \mathrm{~min}$. The transition dose was considered since sources are moved from off position to 4,8 , or $16 \mathrm{~mm}$ position for irradiation and then returned back to off-position after the completion of exposure. Additionally, when sources move from their off position to $16 \mathrm{~mm}$ position, they pass over the $4 \mathrm{~mm}$ collimator holes. Consequently, a very short $4 \mathrm{~mm}$ collimator flash of exposure is delivered in the case of $16 \mathrm{~mm}$ collimator when films are used. This dose for the three apertures was therefore included into the DOR calculation. To determine it, the PTW60019 was used. Two measurements with same beam-on time were performed for all collimator dimensions: in the first measurement, the acquisition started when the sources were in off-position, while in the second one, sources were already in the beam-on position when the electrometer began reading. The difference between the two measurements was considered as transition dose.

\section{B. Measurement setup}

The output ratio measurements were carried out using the ELEKTA spherical dosimetry phantom with a diameter of $16 \mathrm{~cm}$ used for the reference dose-rate calibration of all LGK models. The phantom is constructed from an ABS plastic material. ${ }^{21}$ The phantom has removable inserts that can accommodate different dosimeters including films, ionization chambers, diodes, and other small detectors.

The detectors were placed in the center of the sphere using similar cassettes, which were specifically designed for this purpose. The detectors were oriented in two planes: along the $x-y$ (parallel) plane of the collimator coordinate system (i.e., the $x$-axis is the right-left patient coordinate and the $y$-axis is the posterior-anterior coordinate), and along the $x-z$ (transverse) plane (i.e., the $z$-axis is the cranial-caudal direction) (Fig. 1). The PTW-60019 was mounted in two positions in order to evaluate the geometrical influence of the almost bidimensional

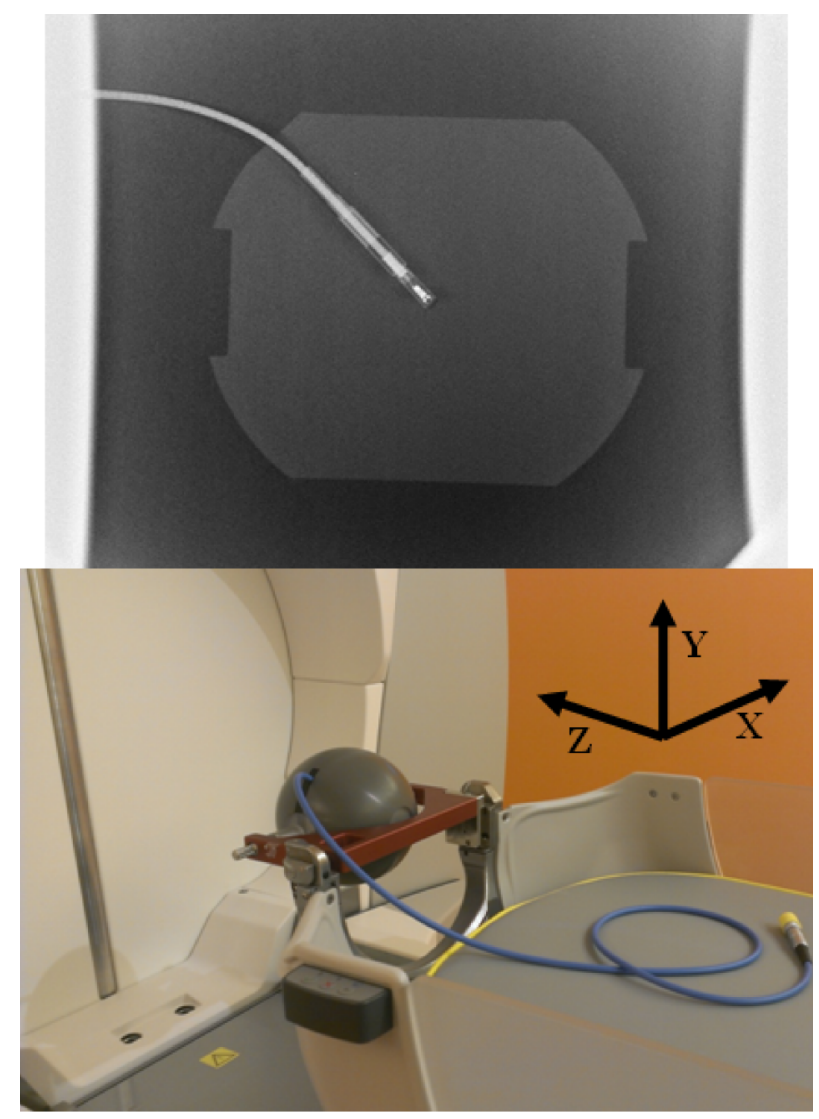

FIG. 1. Spherical dosimetry phantom with holder used for the measurements on LGKP.

diamond active volume (i.e., the diamond diameter is $2 \mathrm{~mm}$, with a few $\mu \mathrm{m}$ thickness) in a 3D irradiation geometry, as the LGK case. The detector cavities allowed the residual errors, though very small, and thus, the exact position of isocenter was determined for each detector and configuration (i.e., transversal and tangential directions) by manual couch motion and profiles acquisition. The profiles were acquired moving the sphere in the three directions. During the measurement of the profiles, the center of the sphere, that is, integral with the detector, is displaced some millimeters from the GK isocenter. An uncertainty in the profile acquisition, especially in the lower penumbra region, arises from this displacement. This uncertainty was evaluated with the TPS and considered in the uncertainty budget. Before this procedure, the couch positioning accuracy was checked to be $<0.1 \mathrm{~mm}$. In detail, LGP was managed in service mode with the possibility to move the couch with $0.1 \mathrm{~mm}$ spacing in the three directions. The Dose1 electrometer (IBA Scanditronix) was used to measure the collected charge and a real-time acquisition was performed with the $4 \mathrm{~mm}$ beams opened. The detector was moved along the three axes up to the maximum reading value, indicating the isocenter position. The maximum vectorial distance from the sphere center (i.e., 100,100,100) was $0.9 \mathrm{~mm}$.

\section{C. Data analysis}

The results were analyzed in terms of dose profiles acquired for the $4 \mathrm{~mm}$ collimator both with PTW-60019 and 

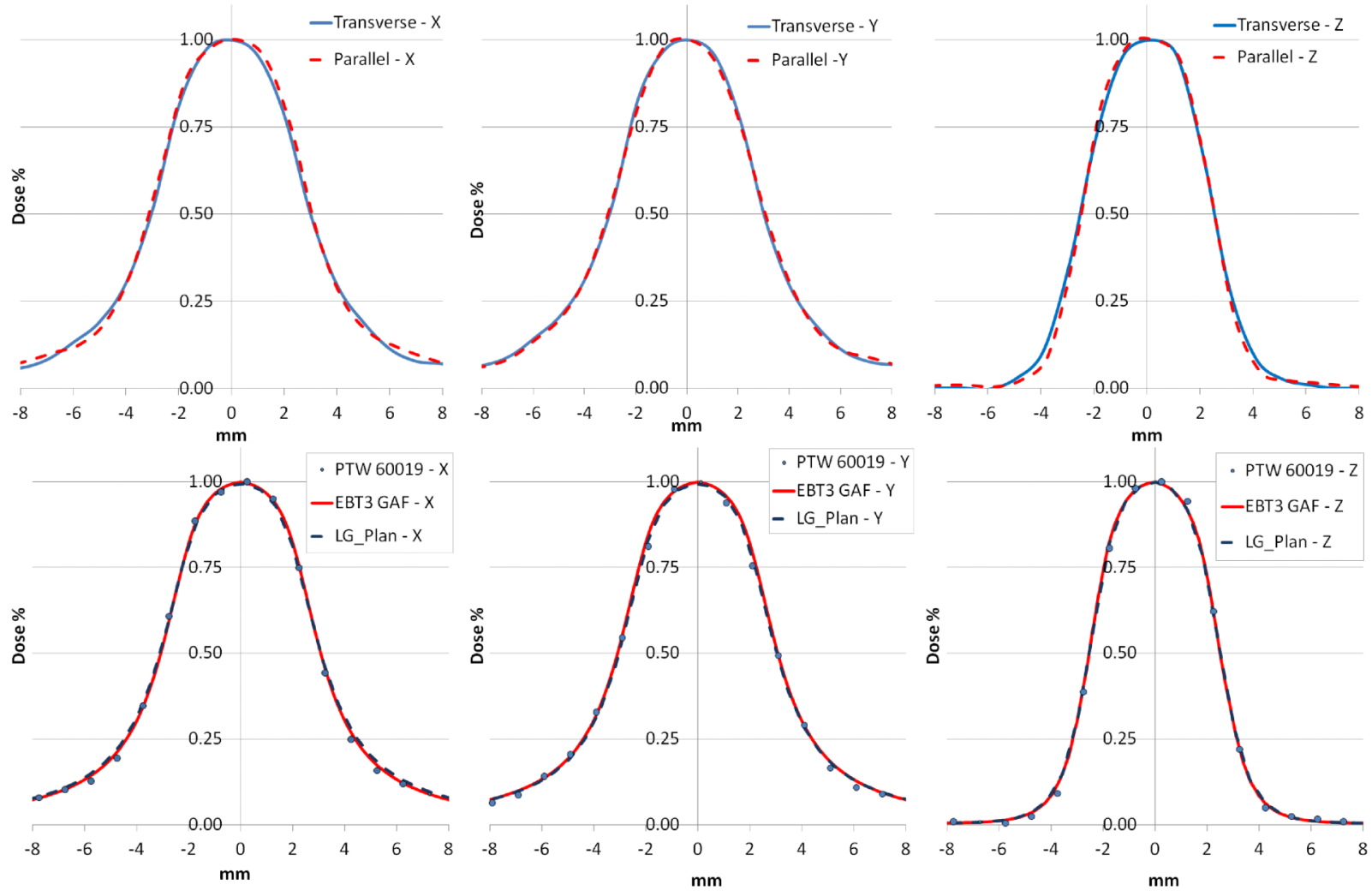

FIG. 2. Profiles along the three axes for the two geometrical configurations of PTW-60019 (top), and for the GafChromic films, LGP, and PTW-60019 (bottom).

GafChromic films in $x, y$, and $z$ directions. The PTW-60019 was positioned in the spherical phantom along parallel and transverse planes and all profiles acquired for each direction. For each profile, the full width at half maximum (FWHM) and the $80 \%-20 \%$ penumbra values were evaluated and compared with the LGP calculations.

DORs were measured with all detectors (PTW-60019, PFD, and SFD) and with GafChromic films for the three collimator sizes. The measurements were normalized to the $16 \mathrm{~mm}$ value. All measurements were performed three times and mean values and standard deviations were then calculated. The MC calculated OFs (LGP 10) were considered as reference values for our measurements. The MC values associated to the old algorithm (LGP 9) were considered for comparison, too. The ratios between the detector specific measured ORs and the MC OFs were calculated for all detectors and compared. These values were referred to as dose response ratio and, as shown by Lechner ${ }^{22}$ and Azangwe, ${ }^{23}$ it can be considered as the reciprocal value of the $k_{Q_{\text {clin }}, Q_{\text {msr }}}^{f_{\text {lin }} f_{\mathrm{mr}}}$ defined by Alfonso et al. ${ }^{3}$

\section{RESULTS}

Profiles of $4 \mathrm{~mm}$ beam in the three directions are reported in Fig. 2 for PTW-60019, GafChromic films, and LGP. The good agreement between the detectors is confirmed by quantitative analysis reported in Table II, in which FWHM and penumbra for the GafChromic films, PTW-60019, SRS, and PFD detectors are measured. As expected, GafChromic films and LGP were in agreement $\leq 0.1 \mathrm{~mm}$ for both FWHM and penumbra. Regarding the PTW-60019, deviations of 0.2 and $0.1 \mathrm{~mm}$ for parallel and transverse directions were found for FWHM. The penumbra was within $0.3 \mathrm{~mm}$ for both parallel and transverse directions.

DORs and dose response ratios are listed in Table III. PTW60019 and SRS detectors in transverse configuration resulted in the best agreement (i.e., <1.6\%) compared to the reference values, and in the parallel configurations, the differences were slightly higher but $\leq 2.6 \%$. In Fig. 3, the dose response ratios are reported for GafChromic films, SFD, and PTW-

TABLE II. FWHM and penumbra for GafChromic films (GAFs), LGP, and PTW-60019 in parallel and transverse directions.

\begin{tabular}{|c|c|c|c|c|c|c|c|c|}
\hline & \multicolumn{4}{|c|}{ FWHM (mm) } & \multicolumn{4}{|c|}{ Penumbra $(\mathrm{mm})$} \\
\hline & GAF & LGP & PTW-60019 parallel & PTW-60019 trans & GAF & LGP & PTW-60019 parallel & PTW-60019 trans \\
\hline $4 \mathrm{~mm}-X$ & 6.1 & 6.1 & 6.2 & 6.1 & 2.7 & 2.8 & 2.7 & 2.9 \\
\hline $4 \mathrm{~mm}-Y$ & 6.1 & 6.1 & 6.3 & 6.1 & 2.7 & 2.8 & 3.0 & 3.0 \\
\hline $4 \mathrm{~mm}-Z$ & 5.0 & 5.0 & 5.0 & 5.1 & 1.6 & 1.5 & 1.6 & 1.9 \\
\hline
\end{tabular}


TABLE III. Detector output ratios (upper part) and dose response ratios (lower part) measured with GafChromic films (i.e., GAF) and with the evaluated detectors (PTW-60019, SRS, and PFD diodes) in both geometrical configurations.

\begin{tabular}{ccccccccccc}
\hline \hline & GAF & PTW-60019 parallel & PTW-60019 transverse & SFD $_{\text {parallel }}$ & SFD $_{\text {transverse }}$ & PFD $_{\text {parallel }}$ & PFD $_{\text {transverse }}$ & LGP 9 & LGP 10 (reference value) \\
\hline $4 \mathrm{~mm}$ & 0.809 & 0.835 & 0.827 & 0.822 & 0.824 & 0.623 & 0.686 & 0.805 & 0.814 \\
$8 \mathrm{~mm}$ & 0.908 & 0.900 & 0.902 & 0.899 & 0.898 & 0.817 & 0.856 & 0.924 & 0.900 \\
\hline $4 \mathrm{~mm}$ & 0.994 & 1.026 & 1.016 & 1.012 & 1.010 & 0.766 & 0.843 & 0.989 & 1.000 \\
$8 \mathrm{~mm}$ & 1.008 & 0.999 & 1.002 & 0.998 & 0.997 & 0.907 & 0.950 & 1.026 & 1.000 \\
\hline \hline
\end{tabular}

60019 detectors in both parallel and transverse configurations. The transition dose was measured with the PTW-60019 and added to the GafChromic films' results. In particular, transition dose was $0.047,0.021$, and $0.014 \mathrm{~Gy}$, for, respectively, 16, 8 , and $4 \mathrm{~mm}$. Without these corrections, the final DORs with GafChromic films would be 0.801 and 0.920 for 4 and $8 \mathrm{~mm}$, respectively.

Overall, the measured collimator dose response ratios show an excellent agreement with the default values used in LGP showing the robustness of the LGP source geometry modeling process. Our results thus validated the new reference values of LGP.

\section{A. Uncertainty estimation}

The positioning uncertainties were estimated to be $0.1 \mathrm{~mm}$ considering the method used to identify the center field and the Gamma Knife mechanical tolerance. The electrometer used for the measurements introduces a further uncertainty of $0.2 \%$, while the statistical dispersion of the repeated measurements introduces a detector-dependent uncertainty ranging from $0.1 \%$ to $0.7 \%$. The overall uncertainties for the DOR measurements were evaluated using the Gaussian error propagation and resulted in an uncertainty $<0.7 \%$ for SFD and PTW60019 (for PFD <1\%). Uncertainties for the measurements performed with GafChromic films were much higher due to the complicated process necessary to perform the measurements and resulted in uncertainties of $0.8 \%, 2.3 \%$, and $3.4 \%$ for the 4,8 , and $16 \mathrm{~mm}$ collimators, respectively. For the profile

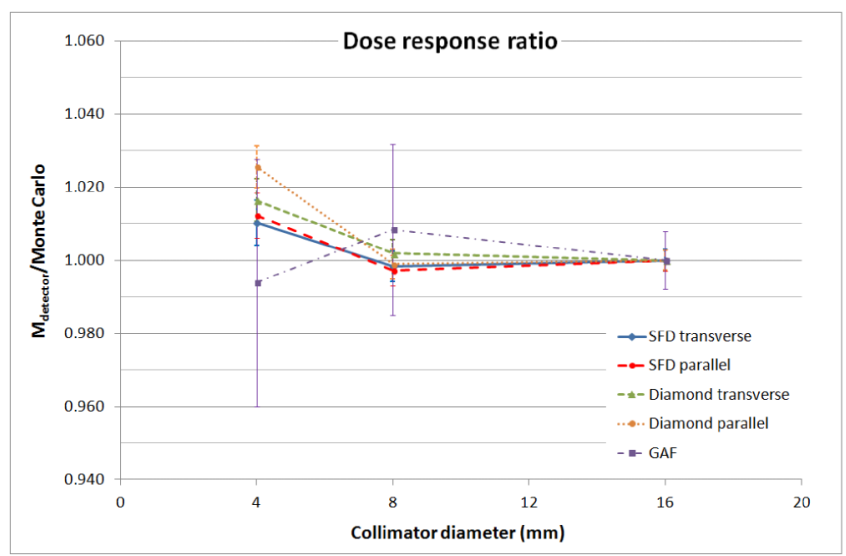

Fig. 3. Dose response ratios for SFD, PTW-60019 (diamond), and GafChromic films (GAFs). The PFD detector was, here, excluded due to its dose underestimation that is likely associated with a large fluence perturbation due to the shield. measurements, a further $0.5 \%$ was added to the uncertainty budget due to the displacement of the spherical phantom from the isocenter during the profile acquisition.

\section{DISCUSSION AND CONCLUSIONS}

The first part of the analysis was focused on the profiles acquired on the three axes in order to evaluate the impact of the finite detectors size on the profile parameters. Figure 2 and the quantitative analysis in Table II revealed the good agreement of the two measurement setups (i.e., parallel and transversal geometries). Furthermore, the good agreement between GafChromic films, LGP, and the diamond detector revealed that both these measurement devices are suitable for profile definition up to the $4 \mathrm{~mm}$ collimators.

Output factor calculation is critical in determining the absolute dose rate for LGK treatments. As an example, in the case of single $4 \mathrm{~mm}$ shot, as the trigeminal neuralgia treatment, the uncertainty in the collimator output factor induces uncertainty in the overall dose delivered, leading to possible different clinical outcomes. ${ }^{24}$

Monte Carlo calculation has been shown as an efficient method in determining OFs. Different authors performed MC calculations of OFs for LGKP and previous models with results mostly consistent with vendor recommended values. ${ }^{25-28}$ These simulations can be used as reference for the OFs calculation. However, actual and independent dosimetric validations are required to check the parameters as part of initial commissioning and routine quality assurance procedures. Each facility is, in fact, responsible for its own dosimetry and output factor values.

Film dosimetry, especially GafChromic film, is a reasonable choice for performing dosimetric measurements in these conditions, since it presents various advantages compared to other detectors. Using film as the measuring medium obviates most of the difficulties encountered in using finite size detectors. ${ }^{20}$ However, taking into account the waiting time to read the films [though with a triple channel analysis, the waiting time could be reduced up to $\sim 1$ h (Ref. 29)], the complexity of the calibration/evaluation process and the cost of consumable material, some institutes can be induced to consider the finite size detectors as an option. When performing measurements with finite detectors in nonstandard fields, the Alfonso formalism should be used. ${ }^{3}$ Since these measurements are not absolute dose measurements, they cannot be strictly considered output factors unless correction factors are applied. In order to be consistent throughout the discussion, the calculated 
ratios will be referred to as detector output ratios even if in the cited papers, these values were called using a different terminology.

The characteristics of these small field dedicated detectors have to be carefully evaluated in order to properly interpret the results obtained in very small field conditions. The uncorrected dose response ratios measured in this work show a slight overresponse for the SFD but a strong underestimation for the PFD with respect to the LGP reference values. The observed values are the result of a volume averaging underestimation on one hand and, on the other hand, an overestimation due to the high density of the materials. As reported in two recent papers, indeed, the physical density of the detector is the dominant parameter in small fields. ${ }^{30,31}$ In large fields, instead, the mass energy absorption coefficient dominates. If no volume averaging were present, there would be an over-response of finite size detectors in such small fields. This effect is largely reported in the literature for diodes ${ }^{22,32}$ and is further enhanced for diamond detectors, due to the higher physical density. The density of the detector casing can also play an important role in small field measurements ${ }^{33}$ though for these three detectors, the materials were quite similar.

The correction factors found in the literature for the PTW microDiamond were compared with the overall correction factors depending both on the material properties of the detector and on the size of the active element. The correction factors resulted $\approx 2 \%$ lower than those proposed by Ralston et al. ${ }^{31}$ both for the 4 and $8 \mathrm{~mm}$ collimator. Azangwe et al ${ }^{23}$ calculated correction factors for different detectors, including PTW-60019, for small fields. Our results are in agreement with theirs within $2.5 \%$ though with slightly lower values. Similar results were obtained comparing the measurements performed by Chalkley and Heyes ${ }^{16}$ on a CyberKnife system. Our dose response ratios thus result in an acceptable agreement with the already published ones, bearing in mind the different conditions in which those results were obtained. In particular, the different conditions in terms of geometry and beam quality affect the correction factors both in the volume averaging component and in the material-related one. The perturbations due to the material density of the diamond detector increase with beam quality ${ }^{34}$ and thus are expected to be lower in a ${ }^{60} \mathrm{Co}$ measurement with respect to the literature $6 \mathrm{MV}$ results. Furthermore, the geometrical conditions of the LGKP (i.e., photons hit the detector from almost all directions) may reduce the volume averaging correction factors. In particular, the influence of the active volume seems to be the dominant parameter at the expense of the active diameter that instead dominates in Linac conditions. This could explain also the difference between PTW-60019 and PFD that is not observed in other Linac-based works. These, however, are hypotheses that should be verified with Monte Carlo methods. In our measurements with the PTW-60019 and the SFD detectors, the two factors, however, compensate each other giving DORs' results very close to the reference ones. In a recent work by Klawikowski et al., ${ }^{35}$ DORs from LGKP were measured with 3D polymer dosimeters and validated by MC measurements. Our results differ from theirs by less than $1.2 \%$ and $0.9 \%$ for the $4 \mathrm{~mm}$ and the $8 \mathrm{~mm}$ collimators, respectively. The correc- tion factors for the PTW-60019 detector on a LGKP device should be further investigated with Monte Carlo methods. The fine quantification of the different components of the correction factors is, indeed, a challenging issue that goes beyond the objectives of the present study.

The GafChromic film measurements show a good agreement with the new reference values with differences $<1 \%$. Our results are in good agreement with the ones obtained by Novotny et al. with EBT films with differences of $0.15 \%$ and $0.9 \%$ for the 4 and $8 \mathrm{~mm}$ collimators, respectively. Novotny et al. showed that GafChromic films seem to be the most suitable choice for these kinds of measurements compared to other films. ${ }^{21}$ In our experience, however, film measurements present the higher uncertainties with respect to microdetectors due both to the different responses between different films and to the complexity of the calibration-measure process.

In conclusion, the PTW-60019 detector is a suitable tool for validating the collimator output factors as part of the initial commissioning and routine quality assurance procedures of LGK though the correction factors have to be deeply investigated. Best results were found with the detector in transverse orientation with a ratio of reading value for the $4 \mathrm{~mm}$ collimator within $1.6 \%$. Based on these measurements, the values of OFs of 0.814 and 0.900 as suggested by ELEKTA were confirmed into the LGP.

\section{ACKNOWLEDGMENTS}

The authors would like to thank Riccardo Pedersini from ELEKTA for the useful discussion and help in performing the measurements. Furthermore, the authors thank Dr. Antonella Del Vecchio from Hospital San Raffaele for the suitable dialogs.

\footnotetext{
a) Author to whom correspondence should be addressed. Electronic mail: giacomo.reggiori@humanitas.it; Telephone: (+39) 02 82248529; Fax: (+39) 0282248509.

${ }^{1}$ C. Lindquist and I. Paddick, "The Leksell Gamma Knife Perfexion and comparisons with its predecessors," Neurosurgery 61, 130-140 (2007).

${ }^{2}$ J. Novotny, J. P. Bhatnagar, A. Niranjan, M. A. Quader, M. S. Huq, G. Bednarz, J. C. Flickinger, D. S. Kondziolka, and L. D. Lunsford, "Dosimetric comparison of the Leksell Gamma Knife Perfexion and 4C,' J. Neurosurg. 109, 8-14 (2008).

${ }^{3}$ R. Alfonso, P. Andreo, R. Capote, M. S. Huq, W. Kilby, P. Kjall, T. R. Mackie, H. Palmans, K. Rosser, J. Seuntjens, W. Ullrich, and S. Vatnitsky, "A new formalism for reference dosimetry of small and nonstandard fields," Med. Phys. 35, 5179-5186 (2008).

${ }^{4}$ M. M. Aspradakis et al., "Small filed MV photon dosimetry," IPEM Report No. 103 (Institute of Physics and Engineering in Medicine, York, UK, 2010).

${ }^{5}$ J. Trnka, J. Novotny, Jr., and J. Kluson, "MCNP-based computational model for the Leksell Gamma Knife,” Med. Phys. 34, 63-75 (2007).

${ }^{6}$ A. Niroomand-Rad et al., "Radiochromic film dosimetry: Recommendations of AAPM Radiation Therapy Committee Task Group 55. American Association of Physicists in Medicine," Med. Phys. 25, 2093-2115 (1998). ${ }^{7}$ S. Suriyapee, N. Pitaxtarnin, S. Oonsiri, C. Jumpangern, I. Israngul, and N. Ayuthaya, "Optimal sensitometric curves of Kodak EDR2 film for dynamic intensity modulated radiation therapy verification," Biomed. Imaging Intervention J. 4, 1-12 (2008).

${ }^{8}$ S. Reinhardt, H. Hillbrand, J. J. Wilkens, and W. Assmann, "Comparison of GafChromic EBT2 and EBT3 films for clinical photon and proton beams," Med. Phys. 39(8), 5257-5262 (2012).

${ }^{9}$ L. Ma, P. Kjäll, J. Novotny, H. Nordström, J. Johansson, and L. Ver-
} hey, "A simple and effective method for validation and measurement of 
collimator output factors for Leksell Gamma Knife Perfexion,” Phys. Med. Biol. 54(12), 3897-3907 (2009).

${ }^{10}$ H. Bilge, Z. Osen, O. Senkesen, H. Kucucuk, A. Cakir, and M. Sengoz, "Determination of output factors for the Leksell Gamma Knife using ion chamber, thermoluminescence detectors and films," J. Buon. 11, 223-227 (2006).

${ }^{11}$ L. Ma, X. A. Li, and C. X. Yu, "An efficient method of measuring the $4 \mathrm{~mm}$ output factor for the Gamma Knife," Phys. Med. Biol. 45, 729-733 (2000).

${ }^{12}$ A. Mack, S. G. Scheib, J. Major, S. Gianolini, G. Pazmandi, H. Feist, H. Czempiel, and H. J. Kreiner, "Precision dosimetry for narrow photon beams used in radiosurgery-determination of Gamma Knife output factors," Med. Phys. 29, 2080-2089 (2002).

${ }^{13}$ J. S. Tsai, M. J. Rivard, M. J. Engler, J. E. Mignano, D. E. Wazer, and W. A. Shucart, "Determination of the $4 \mathrm{~mm}$ Gamma Knife helmet relative output factor using a variety of detectors," Med. Phys. 30, 986-992 (2003).

${ }^{14}$ C. Yu, G. Luxton, M. L. Apuzzo, and Z. Petrovich, "TLD measurements of the relative output factors for the Leksell Gamma Knife," Stereotact. Funct. Neurosurg. 72(Suppl. 1), 150-158 (1999).

${ }^{15}$ I. Ciancaglioni et al., "Dosimetric characterization of a synthetic single crystal diamond detection in clinical radiation therapy small photon beams," Med. Phys. 39, 4493-4501 (2012).

${ }^{16} \mathrm{~A}$. Chalkley and G. Heyes, "Evaluation of a synthetic single-crystal diamond detector for relative dosimetry measurements on a CyberKnife," Br. J. Radiol. 87, 20130768 (2014).

${ }^{17}$ A. K. Mandapaka, A. Ghebremedhin, B. Patyal, M. Marinelli, G. Prestopino, C. Verona, and G. Verona-Rinati, "Evaluation of the dosimetric properties of a synthetic single crystal diamond detector in high energy clinical proton beams," Med. Phys. 40(12), 121702 (9pp.) (2013).

${ }^{18} \mathrm{M}$. Zani et al., "A synthetic diamond diode in volumetric modulated arc therapy dosimetry," Med. Phys. 40, 092103 (7pp.) (2013).

${ }^{19} \mathrm{~F}$. Marsolat et al., "Diamond dosimeter for small beam stereotactic radiotherapy," Diamond Relat. Mater. 33, 53-70 (2013).

${ }^{20}$ B. C. Ferreira, M. C. Lopes, and M. Capela, "Evaluation of an Epson flatbed scanner to read GafChromic EBT films for radiation dosimetry," Phys. Med. Biol. 54, 1073-1085 (2009).

${ }^{21}$ J. Novotny, Jr., J. P. Bhatnagar, and M. A. Quader, "Measurement of relative output factors for the 8 and $4 \mathrm{~mm}$ collimators of Leksell Gamma Knife Perfexion by film dosimetry," Med. Phys. 36, 1768-1774 (2009).

${ }^{22}$ W. Lechner, H. Palmans, L. Sölkner, P. Grochowska, and D. Georg, "Detector comparison for small field output factor measurements in flattening filter free photon beams," Radiother. Oncol. 109, 356-360 (2013).
${ }^{23} \mathrm{G}$. Azangwe et al., "Detector to detector corrections: A comprehensive experimental study of detector specific correction factors for beam output measurements for small radiotherapy beams," Med. Phys. 41, 072103 (16pp.) (2014)

${ }^{24}$ N. Massager, N. Murata, M. Tamura, D. Devriendt, M. Levivier, and J. Régis, "Influence of nerve radiation dose in the incidence of trigeminal dysfunction after trigeminal neuralgia radiosurgery," Neurosurgery 60, 681-687 (2007).

${ }^{25}$ G. Battistoni, F. Cappucci, N. Bertolino, M. G. Brambilla, H. S. Mainardi, and A. Torresin, "FLUKA Monte Carlo simulation for the Leksell Gamma Knife Perfexion radiosurgery system: Homogeneous media," Phys. Med. 29(6), 656-661 (2013).

${ }^{26}$ Y. C. Cheung, K. N. Yu, C. P. Yu, and R. T. K. Ho, "Monte Carlo calculated output factors of a Leksell Gamma Knife unit," Phys. Med. Biol. 44, N247-N249 (1999).

${ }^{27}$ V. Moskvin, C. DesRosiers, L. Papiez, R. Timmerman, M. Randall, and P. DesRosiers, "Monte Carlo simulation of the Leksell Gamma Knife: I. Source modeling and calculation in homogeneous media," Phys. Med. Biol. 47, 1995-2011 (2002).

${ }^{28}$ V. Casanova Borca et al., "Dosimetric characterization and use of GAFCHROMIC EBT3 film for IMRT dose verification," J. Appl. Clin. Med. Phys. 14, 158-171 (2013).

${ }^{29}$ A. Micke, D. F. Lewis, and X. Yu, "Multichannel film dosimetry with nonuniformity correction,” Med. Phys. 38(5), 2523-2534 (2011).

${ }^{30}$ A. J. Scott, S. Kumar, A. E. Nahum, and J. D. Fenwick, "Characterizing the influence of detector density on dosimeter response in non-equilibrium small photon fields," Phys. Med. Biol. 57, 4461-4476 (2012).

${ }^{31}$ A. Ralston, M. Tyler, P. Liu, D. McKenzie, and N. Suchowerska, "Overresponse of synthetic microDiamond detectors in small radiation fields," Phys. Med. Biol. 59, 5873-5881 (2014).

${ }^{32}$ A. Ralston, P. Liu, K. Warrener, D. McKenzie, and N. Suchowerska, "Small field diode correction factors derived using an air core fibre optic scintillation dosimeter and EBT2 film," Phys. Med. Biol. 57, 2587-2602 (2012).

${ }^{33}$ T. S. Underwood, H. C. Winter, M. A. Hill, and J. D. Fenwick, "Mass-density compensation can improve the performance of a range of different detectors under non-equilibrium conditions," Phys. Med. Biol. 58, 8295-8310 (2013).

${ }^{34}$ M. Pimpinella, I. Ciancaglioni, R. Consorti, C. Di Venanzio, A. S. Guerra, A. Petrucci, A. Stravato, and G. Verona-Rinati, "A synthetic diamond detector as transfer dosimeter for $D_{\mathrm{w}}$ measurements in photon beams with small field sizes," Metrologia 49, S207-S210 (2012).

${ }^{35}$ S. J. Klawikowski, J. N. Yang, J. Adamovics, and G. S. Ibbott, "PRESAGE 3D dosimetry accurately measures Gamma Knife output factors," Phys. Med. Biol. 59, N211-N220 (2014). 